\title{
Predicting the chemosensitivity of pancreatic cancer cells by quantifying the expression levels of genes associated with the metabolism of gemcitabine and 5-fluorouracil
}

\author{
NOBUAKI KURATA ${ }^{1}$, HAYATO FUJITA ${ }^{1}$, KENOKI OHUCHIDA ${ }^{1,2}$, KAZUHIRO MIZUMOTO ${ }^{1,3}$, \\ PRAWEJ MAHAWITHITWONG ${ }^{1}$, HIROSHI SAKAI ${ }^{1}$, MANABU ONIMARU ${ }^{1}$, TATSUYA MANABE ${ }^{1}$, \\ TAKAO OHTSUKA $^{1}$ and MASAO TANAKA ${ }^{1}$
}

Departments of ${ }^{1}$ Surgery and Oncology, and ${ }^{2}$ Advanced Medical Initiatives, Graduate School of Medical Sciences, Kyushu University; ${ }^{3}$ Kyushu University Hospital Cancer Center, 3-1-1 Maidashi, Fukuoka 812-8582, Japan

Received March 1,2011; Accepted April 27, 2011

DOI: 10.3892/ijo.2011.1058

\begin{abstract}
Gemcitabine (GEM) is the standard treatment for advanced/metastatic pancreatic cancer. However, there is a substantial subset of patients in whom the efficacy of GEM, when used as a single agent, is inadequate. Recently, the 5-fluorouracil (5-FU) prodrugs capecitabine and S-1 have been used as an alternative, either alone or in combination with GEM. The aim of the present study was to investigate the expression pattern of genes that render pancreatic cancer cells sensitive to GEM and 5-FU, and to identify markers for individualized chemotherapy, even in patients who have developed resistance. We investigated the correlation between the expression of genes associated with the metabolism of GEM and 5-FU, and sensitivity to these drugs in 15 human pancreatic cancer cell lines. We also established GEM- and 5-FU-resistant pancreatic cancer cell lines to investigate changes in the expression levels of these genes and the effects of one drug on cells resistant to the other. We found no correlation between pancreatic cancer cell sensitivity to either GEM- or 5-FU. GEM-resistant cells did not become resistant to 5-FU and vice versa. High expression of $R R M 1(\mathrm{P}=0.048)$ and $T S \times D P D$ $(\mathrm{P}=0.035)$ correlated significantly with sensitivity to GEM and 5-FU, respectively. 5-FU-resistant cells expressed significantly higher levels of $T P$ than parental cells $(\mathrm{P}<0.05)$. In conclusion, pancreatic cancer cells showed no crossresistance to GEM and 5-FU. Quantitative analyses of $R R M 1$, $T P, D P D$ and $T S$ mRNA levels in pancreatic cancer cells may be useful for predicting their sensitivity to GEM and 5-FU.
\end{abstract}

Correspondence to: Dr Hayato Fujita or Dr Kazuhiro Mizumoto, Department of Surgery and Oncology, Graduate School of Medical Sciences, Kyushu University, 3-1-1 Maidashi, Fukuoka 812-8582, Japan

E-mail: hayato@surg1.med.kyushu-u.ac.jp

E-mail:mizumoto@med.kyushu-u.ac.jp

Key words: gemcitabine, 5-fluorouracil, reverse transcriptionpolymerase chain reaction, chemosensitivity, pancreatic cancer

\section{Introduction}

The prognosis for patients with pancreatic cancer is extremely poor. The tumor is extremely aggressive and early detection is difficult due to the lack of early disease-specific signs and symptoms. Only $10-20 \%$ of patients with pancreatic cancer are candidates for curative resection $(1,2)$ and, even if surgery is performed, the post-operative 5-year survival rate is only $15-25 \%$ due to the high incidence of postoperative recurrence $(1,3,4)$. Gemcitabine (difluorodeoxycitidine, dFdC; GEM) is the standard treatment for advanced/metastatic pancreatic cancer based on a landmark trial comparing its effects with those of fluorouracil (FU) (5). However, the clinical benefit of GEM as a single agent is inadequate, as indicated by the median survival time of only 5.7-7.2 months and a low objective response rate (5-9). Thus, there is a pressing need to develop new treatment strategies. Recently, a phase III trial for advanced pancreatic cancer showed a significant increase in both overall survival (OS) and progression-free survival (PFS) after treatment with erlotinib plus GEM compared with GEM alone. Although these results were statistically significant, the absolute benefit of OS was modest (only 2 weeks) (9).

GEM is a deoxycytidine analog that has significant singleagent activity against a number of malignancies, including pancreatic cancer $(10,11)$. GEM is transported into cells via the human equilibrative nucleoside transporter-1 (hENT1) (12) and must be phosphorylated by deoxycytidine kinase (dCK) to be activated. The phosphorylated forms of GEM inhibit DNA synthesis through its incorporation into DNA leading to masked chain termination, and by inhibiting the enzyme ribonucleotide reductase (RR) $(13,14)$. In addition, the deoxyribonucleotide and ribonucleotide pools, both essential for DNA repair, are seriously depleted by the phosphorylated forms (15). Conversely, GEM is inactivated by cytidine deaminase (CDA) (16). We, and other investigators, showed that the expressions of hENT1, dCK, the RR subunits M1 (RRM1) and M2 (RRM2), and the genes that encode them were, at least partially, correlated with sensitivity to GEM (17-25).

Recently, the orally administered fluoropyrimidine prodrugs, capecitabine and S-1, have been used as alternative 
Table I. Primer sequences and product sizes.

\begin{tabular}{|c|c|c|c|}
\hline Primer & Forward sequence $5^{\prime}-3^{\prime}$ & Reverse sequence 5'-3' & Product size \\
\hline hENT1 & gcaaaggagaggagccaagag & gggctgagagttggagactg & 65 \\
\hline$d C K$ & gctgcagggaagtcaacattt & ttcaggaaccacttcccaatc & 69 \\
\hline$R R M 1$ & actaagcaccetgactatgctatcc & cttcctcacatcactgaacacttt & 88 \\
\hline$R R M 2$ & ggctcaagaaacgaggactg & tcaggcaagcaaaatcacag & 93 \\
\hline$C D A$ & tcaaagggtgcaacatagaaaatg & cggtccgttcagcacagat & 61 \\
\hline$T P$ & cctgcggacggaatcct & gctgtgatgagtggcaggct & 71 \\
\hline$D P D$ & aggacgcaaggagggtttg & gtccgecgagtccttactga & 84 \\
\hline OPRT & tcctgggcagatctagtaaatgg & tgetcctcagccattctaacc & 156 \\
\hline$T S$ & gcctcggtgtgcctttca & cccgtgatgtgcgcaat & 67 \\
\hline $18 S r R N A$ & gtaacccgttgaaccccatt & ccatccaatcggtagtagcg & 151 \\
\hline
\end{tabular}

or additional agents for advanced pancreatic cancer. A recent phase III clinical trial for advanced pancreatic cancer showed that treatment with GEM plus capecitabine led to a significant increase in PFS and a tendency to prolonged OS compared with GEM alone (7). Capecitabine is metabolized to 5-FU via a three-step enzymatic process, the final step being catalyzed by thymidine phosphorylase (TP) (26). Meanwhile, a late phase II study using S-1 to treat metastatic pancreatic cancer showed promising results, with a $37.5 \%$ response rate and a median OS of 9.2 months (27). S-1 consists of tegafur (FT; a prodrug of 5-FU) and two biochemical modulators, 5-chloro2,4-dihydroxypyridine (CDHP) and potassium oxonate (Oxo) (28), which improve the tumor-selective toxicity of 5-FU. CDHP is a competitive inhibitor of dihydropyrimidine dehydrogenase (DPD), which rapidly catabolizes 5-FU and maintains efficacious 5-FU concentrations in the plasma and tumor tissues (29). Oxo decreases phosphorylation of 5-FU within the gastrointestinal tract by competitively inhibiting orotate phosphoribosyltransferase (OPRT), thereby reducing the serious gastrointestinal toxicity associated with 5-FU (30). Finally, in both agents, 5-FU interacts with its pharmacological target, thymidylate synthase (TS) and inhibits DNA synthesis and repair. Increasing evidence suggests that the expression levels of TP, DPD, OPRT and TS (along with the genes that encode them) predict sensitivity to 5-FU or its prodrugs (26,29-32).

Although GEM and 5-FU prodrugs are effective against advanced pancreatic cancer when used as single agents, there is a substantial subset of patients in whom the efficacy is limited or inadequate. Also, few studies have investigated whether these agents are effective in patients who have developed resistance to other agents. Recent studies show that altered gene expression can, at least in part, explain the efficacy of cytotoxic agents $(19,33)$. Therefore, in the present study, we investigated the correlation between the expression of genes associated with the metabolism of GEM and 5-FU and cancer cell sensitivity to the drugs using 15 human pancreatic cancer cell lines. Furthermore, we established pancreatic cancer cell lines that are resistant to each agent to investigate the effects of one drug on pancreatic cancer cell lines that were resistant to the other. We also analyzed the expression levels of genes related to the transport and metabolism of GEM and 5-FU to clarify the underlying mechanisms involved in drug-resistance.

\section{Materials and methods}

Cell lines and establishment of GEM or 5-FU-resistant cells. The following 15 human pancreatic cancer cell lines were used in this study: BxPC-3, Capan-1, Capan-2, CFPAC1, Hs766T, SW1990 (American Type Culture Collection, Manassas, Virginia, USA), AsPC-1, H48N, KP-1N, KP-2, KP-3, Panc-1, SUIT-2 (generously provided by Dr H. Iguchi (National Shikoku Cancer Center, Matsuyama, Japan), MIA PaCa-2 (Japanese Cancer Resources Bank, Tokyo, Japan) and NOR-P1; established in our laboratory (34). Cells were maintained as previously described (35). Cells resistant to GEM (Wako, Osaka, Japan) or 5-FU (Kyowa Hakko Kogyo, Tokyo, Japan) were generated by exposure to gradually increasing concentrations of each drug as previously described (23). The final concentrations of GEM and 5-FU were $200 \mathrm{nM}$ and $2 \mu \mathrm{M}$, respectively. Both agents were dissolved in phosphate-buffered saline and added to the culture medium [Dulbecco's modified Eagle's medium, DMEM; Sigma Chemical Co., St. Louis, MO, USA; supplemented with $10 \%$ fetal bovine serum (FBS), streptomycin $(100 \mu \mathrm{g} / \mathrm{ml})$ and penicillin $(100 \mathrm{U} / \mathrm{ml})]$.

Propidium iodide (PI) assay. To calculate the $50 \%$ inhibitory concentration $\left(\mathrm{IC}_{50}\right)$ for each cell line when exposed to GEM or 5-FU, cells were seeded in 24-well plates (BectonDickinson Labware, Bedford, MA, USA) at a density of $2 \times 10^{4}$ per well, using cell numbers previously counted using a particle distribution analyzer (CDA 500; Sysmex, Kobe, Japan). Several different concentrations of GEM or 5-FU were added to the cells $24 \mathrm{~h}$ after seeding. Cell populations were evaluated by measuring the fluorescence intensity of PI after a further incubation for $72 \mathrm{~h}$, as previously described $(19,23)$.

Quantitative one-step real-time reverse transcriptionpolymerase chain reaction $(q R T-P C R)$. Total RNA was extracted from cultured cells using a High Pure RNA isolation kit (Roche, Mannheim, Germany) with DNase (Roche) treatment according to the manufacturer's instructions. qRT-PCR was performed for 40 cycles of $15 \mathrm{sec}$ at $95^{\circ} \mathrm{C}$ and $1 \mathrm{~min}$ at $55^{\circ} \mathrm{C}$ using a Chromo 4 real-time PCR detection system (Bio-Rad Laboratories, Hercules, CA, USA) and a 
Table II. $\mathrm{IC}_{50}$ values for each of the pancreatic cancer cell lines.

\begin{tabular}{lrc}
\hline & \multicolumn{2}{c}{ IC $_{50}$ value } \\
\cline { 2 - 3 } Cell line & GEM $(\mathrm{nM})$ & 5 -FU $(\mu \mathrm{M})$ \\
\hline AsPC-1 & 17.0 & 3.25 \\
BxPC-3 & 17.9 & 6.29 \\
Capan-1 & 62.6 & 2.35 \\
Capan-2 & 307.0 & 2.89 \\
CFPAC1 & 328.0 & 2.52 \\
H48N & 24.0 & 0.93 \\
Hs766T & 314.0 & 1.20 \\
KP-1N & 524.3 & 3.76 \\
KP-2 & 435.3 & 2.50 \\
KP-3 & 4.7 & 1.00 \\
MIA PaCa-2 & 51.0 & 9.00 \\
NOR-P1 & 304.0 & 2.97 \\
Panc1 & 27.0 & 3.85 \\
SUIT-2 & 3.5 & 4.01 \\
SW1990 & 270.7 & 5.68 \\
\hline
\end{tabular}

QuantiTect SYBR-Green reverse transcription-PCR kit (Qiagen) according to the manufacturer's instructions (36). Specific primers were designed (Table I), and BLAST searches were performed to ensure the primer specificities. The levels of $h E N T 1, d C K, R R M 1, R R M 2, T P, O P R T$ and TS mRNA were calculated from standard curves constructed using total RNA from Capan-1 cells. The levels of $C D A$ and $D P D$ mRNA were calculated from standard curves constructed using total RNA from SUIT-2 cells. The level of each mRNA was normalized to that of $18 S$ rRNA.

Statistical analysis. Statistical analyses and graphical presentations were done using JMP 8.0 software (SAS Institute, Cary, $\mathrm{NC}$, USA). Values were expressed as the mean \pm SD. Comparisons between two groups were done using Student's t-test. The correlation between two groups was analyzed using Spearman's rank-correlation test. Statistical significance was defined as $\mathrm{P}<0.05$.

\section{Results}

Correlation between GEM or 5-FU IC ${ }_{50}$ values and gene expression levels associated with drug metabolism. To investigate the chemosensitivity of pancreatic cancer cells to GEM and 5-FU, the $\mathrm{IC}_{50}$ values after exposure were calculated for all 15 cell lines (Table II). There was no significant correlation between the $\mathrm{IC}_{50}$ values for GEM and 5-FU in these cell lines [Fig. 1A; Spearman's rank-correlation coefficient (Q): $-0.16, \mathrm{P}=0.57]$.

We next quantified the expression levels of genes involved in the cellular uptake and metabolism of GEM and 5-FU (Tables III and IV) and analyzed the correlation between the $\mathrm{IC}_{50}$ values for these agents and the expression level of each gene (Tables $\mathrm{V}$ and VI). The results showed a significant
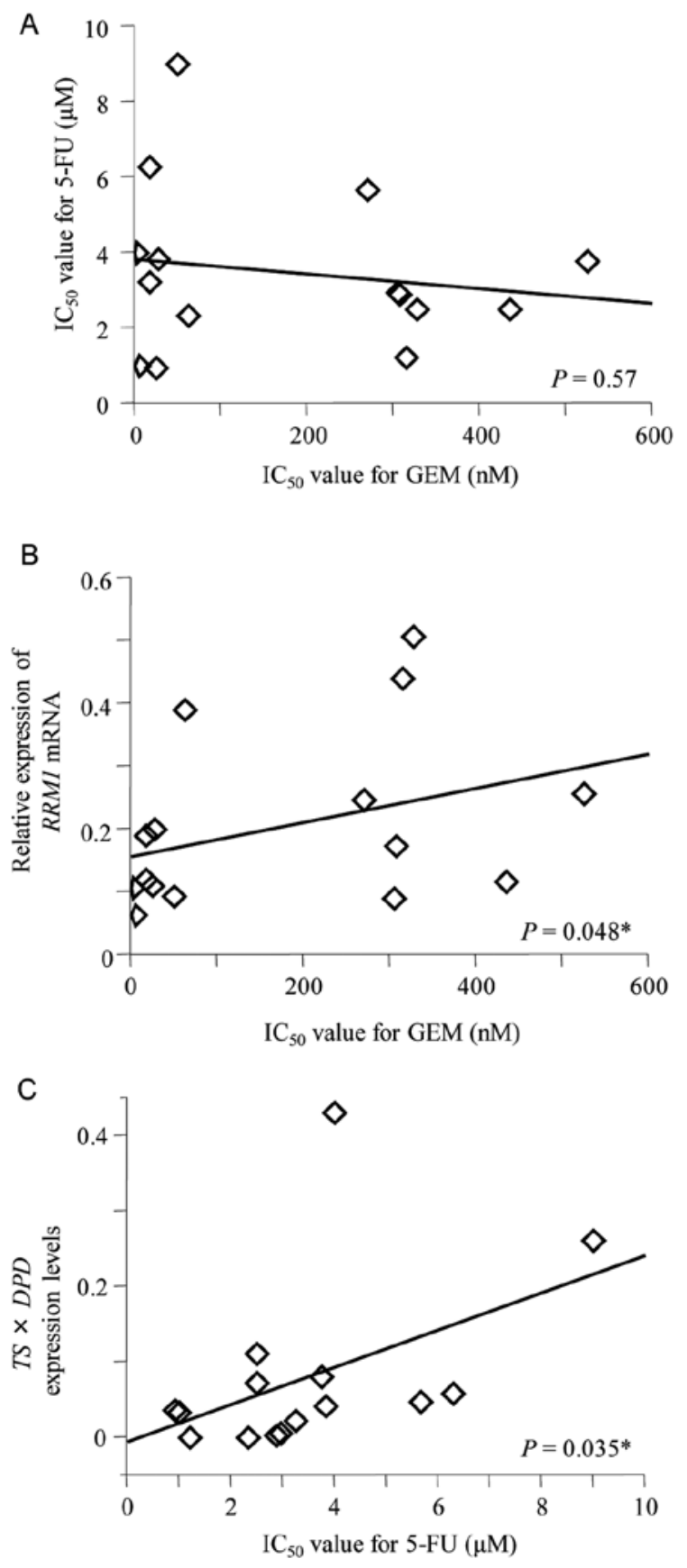

Figure 1. There was no significant correlation between the $\mathrm{IC}_{50}$ values for GEM and 5-FU in 15 pancreatic cancer cell lines [Spearman's rank-correlation coefficient $(\mathrm{Q})$ : $-0.16, \mathrm{P}=0.57](\mathrm{A})$. There was a significant correlation between $\mathrm{GEM} \mathrm{IC}_{50}$ values and $R R M 1$ expression levels [Spearman's rankcorrelation coefficient (Q): $0.52, \mathrm{P}=0.048](\mathrm{B})$. There was a significant correlation between 5-FU $\mathrm{IC}_{50}$ values and $T S \times D P D$ expression levels [Spearman's rank-correlation coefficient (@): 0.55, $\mathrm{P}=0.035](\mathrm{C}) .{ }^{*} \mathrm{P}<0.05$.

correlation between the $\mathrm{IC}_{50}$ values for GEM and RRMI expression levels [Fig. 1B and Table V; Spearman's rankcorrelation coefficient (@): $0.52, \mathrm{P}=0.048$ ], suggesting that pancreatic cancer cells with high $R R M 1$ expression levels were more resistant to GEM. Although the ratio of $h E N T 1 \mathrm{x}$ $d C K / R R M 1 \times R R M 2$ expression is thought to be useful for predicting GEM- sensitivity in pancreatic cancer cells (37), we found no significant correlation [Table V; Spearman's rank-correlation coefficient $(\varrho)$ : $-0.27, \mathrm{P}=0.33]$. In addition, 
Table III. Relative expression levels of mRNAs associated with GEM metabolism in pancreatic cancer cell lines.

\begin{tabular}{lccccc}
\hline & \multicolumn{5}{c}{ Relative mRNA expression levels normalized to those of $18 S$ rRNA } \\
\cline { 2 - 6 } Cell line & $h E N T 1$ & $d C K$ & $R R M 1$ & $R R M 2$ & CDA \\
\hline AsPC-1 & $0.079 \pm 0.0083$ & $0.150 \pm 0.0140$ & $0.190 \pm 0.085000$ & $0.190 \pm 0.0170$ & $10.01 \pm 1.7000$ \\
BxPC-3 & $0.062 \pm 0.0079$ & $0.190 \pm 0.0033$ & $0.120 \pm 0.037000$ & $0.160 \pm 0.0040$ & $3.700 \pm 0.6700$ \\
Capan-1 & $0.180 \pm 0.0012$ & $0.360 \pm 0.0710$ & $0.390 \pm 0.080000$ & $0.450 \pm 0.0230$ & $0.043 \pm 0.0084$ \\
Capan-2 & $0.091 \pm 0.0039$ & $0.190 \pm 0.0390$ & $0.170 \pm 0.007700$ & $0.270 \pm 0.0210$ & $0.201 \pm 0.0630$ \\
CFPAC1 & $0.130 \pm 0.0021$ & $0.120 \pm 0.0260$ & $0.510 \pm 0.160000$ & $0.350 \pm 0.0220$ & $5.150 \pm 0.1400$ \\
H48N & $0.100 \pm 0.0061$ & $0.052 \pm 0.0270$ & $0.110 \pm 0.000690$ & $0.250 \pm 0.0110$ & $1.430 \pm 0.2500$ \\
Hs766T & $0.083 \pm 0.0067$ & $0.240 \pm 0.0100$ & $0.440 \pm 0.110000$ & $0.200 \pm 0.0067$ & $0.039 \pm 0.0087$ \\
KP-1N & $0.410 \pm 0.0340$ & $0.230 \pm 0.0390$ & $0.260 \pm 0.051000$ & $0.600 \pm 0.0350$ & $0.056 \pm 0.0150$ \\
KP-2 & $0.046 \pm 0.0030$ & $0.099 \pm 0.0042$ & $0.120 \pm 0.054000$ & $0.092 \pm 0.0046$ & $0.550 \pm 0.1100$ \\
KP-3 & $0.055 \pm 0.0066$ & $0.150 \pm 0.0074$ & $0.064 \pm 0.000046$ & $0.270 \pm 0.0098$ & $0.620 \pm 0.1200$ \\
MIA PaCa-2 & $0.190 \pm 0.0031$ & $0.074 \pm 0.0092$ & $0.094 \pm 0.013000$ & $0.240 \pm 0.0021$ & $4.780 \pm 3.0800$ \\
NOR-P1 & $0.140 \pm 0.0096$ & $0.130 \pm 0.0190$ & $0.089 \pm 0.029000$ & $0.290 \pm 0.0011$ & $0.260 \pm 0.0220$ \\
Panc1 & $0.330 \pm 0.0061$ & $0.091 \pm 0.0028$ & $0.200 \pm 0.007600$ & $0.260 \pm 0.0190$ & $0.039 \pm 0.0067$ \\
SUIT-2 & $0.140 \pm 0.0058$ & $0.320 \pm 0.0800$ & $0.110 \pm 0.005000$ & $0.410 \pm 0.0022$ & $0.690 \pm 0.2000$ \\
SW1990 & $0.074 \pm 0.0043$ & $0.120 \pm 0.0043$ & $0.250 \pm 0.031000$ & $0.280 \pm 0.0150$ & $6.420 \pm 0.9200$ \\
\hline
\end{tabular}

Table IV. Relative expression levels of mRNAs associated with 5-FU metabolism in the pancreatic cancer cell lines.

\begin{tabular}{lcccc}
\hline & \multicolumn{4}{c}{ Relative mRNA expression levels normalized to those of $18 S$ r $R N A$} \\
\cline { 2 - 5 } Cell line & $T P$ & $D P D$ & OPRT & $T S$ \\
\hline AsPC-1 & $2.80 \pm 0.440$ & $0.2600 \pm 0.00099$ & $0.088 \pm 0.000073$ & $0.087 \pm 0.0091$ \\
BxPC-3 & $7.80 \pm 4.350$ & $0.3500 \pm 0.04200$ & $0.077 \pm 0.004000$ & $0.170 \pm 0.0240$ \\
Capan-1 & $0.41 \pm 0.140$ & $0.0023 \pm 0.00056$ & $0.073 \pm 0.002500$ & $0.250 \pm 0.0420$ \\
Capan-2 & $1.40 \pm 0.390$ & $0.0200 \pm 0.00180$ & $0.062 \pm 0.002100$ & $0.130 \pm 0.0270$ \\
CFPAC1 & $0.18 \pm 0.019$ & $0.3000 \pm 0.07000$ & $0.049 \pm 0.000043$ & $0.240 \pm 0.0430$ \\
H48N & $6.64 \pm 3.770$ & $0.2900 \pm 0.02000$ & $0.110 \pm 0.002200$ & $0.120 \pm 0.0110$ \\
Hs766T & $0.22 \pm 0.077$ & $0.0047 \pm 0.00230$ & $0.083 \pm 0.014000$ & $0.170 \pm 0.0057$ \\
KP-1N & $1.22 \pm 0.150$ & $0.2800 \pm 0.02300$ & $0.068 \pm 0.000530$ & $0.290 \pm 0.0330$ \\
KP-2 & $0.67 \pm 0.044$ & $0.6300 \pm 0.04600$ & $0.038 \pm 0.004100$ & $0.180 \pm 0.0340$ \\
KP-3 & $0.87 \pm 0.050$ & $0.4300 \pm 0.01500$ & $0.057 \pm 0.010000$ & $0.075 \pm 0.0019$ \\
MIA PaCa-2 & $0.19 \pm 0.034$ & $1.0900 \pm 0.07400$ & $0.130 \pm 0.020000$ & $0.240 \pm 0.0072$ \\
NOR-P1 & $1.86 \pm 0.130$ & $0.0340 \pm 0.00810$ & $0.061 \pm 0.007000$ & $0.150 \pm 0.0078$ \\
Panc1 & $1.06 \pm 0.800$ & $0.3300 \pm 0.05500$ & $0.053 \pm 0.001600$ & $0.130 \pm 0.0041$ \\
SUIT-2 & $1.77 \pm 1.140$ & $0.9700 \pm 0.06300$ & $0.110 \pm 0.002600$ & $0.440 \pm 0.0400$ \\
SW1990 & $0.39 \pm 0.061$ & $0.2600 \pm 0.03100$ & $0.033 \pm 0.000250$ & $0.180 \pm 0.0790$ \\
\hline
\end{tabular}

there was no significant correlation between the $\mathrm{IC}_{50}$ values for GEM and the expression of genes associated with 5-FU metabolism (data not shown). Also, there was no significant correlation between the $\mathrm{IC}_{50}$ values for $5-\mathrm{FU}$ and the expression levels of these genes (Table VI). However, a significant correlation was found between the $\mathrm{IC}_{50}$ values for 5-FU and the expression of TS x DPD [Fig. 1C and Table VI; Spearman's rank-correlation coefficient (@): $0.55, \mathrm{P}=0.035]$, suggesting that pancreatic cancer cells with high $T S$ and/or
$D P D$ expression levels were more resistant to 5-FU. Although the ratios of $T P / D P D$ and $O P R T / D P D$ were reported to be useful for predicting patients' prognosis or sensitivity to 5-FU-based chemotherapy $(38,39)$, we found no significant correlation [Table VI; Spearman's rank-correlation coefficient (Q): -0.38 and $-0.44, \mathrm{P}=0.16$ and 0.10 , respectively]. There was also no significant correlation between the $\mathrm{IC}_{50}$ values for 5-FU and the expression of genes associated with GEM metabolism (data not shown). Although RR is reported to 
Table V. Correlation between the $\mathrm{IC}_{50}$ values for GEM and the mRNA expression levels of genes associated with GEM metabolism.

\begin{tabular}{|c|c|c|}
\hline Gene expressions & $\begin{array}{l}\text { Spearman's rank- } \\
\text { correlation } \\
\text { coefficient (@) }\end{array}$ & P-value \\
\hline hENT1 & 0.12 & 0.67 \\
\hline$d C K$ & -0.025 & 0.93 \\
\hline$R R M 1$ & 0.52 & $0.048^{\mathrm{a}}$ \\
\hline$R R M 2$ & 0.14 & 0.63 \\
\hline$C D A$ & -0.34 & 0.21 \\
\hline$h E N T 1 \times d C K$ & 0.18 & 0.52 \\
\hline$R R M 1 \times R R M 2$ & 0.40 & 0.14 \\
\hline$h E N T 1 \times d C K /$ RRM1 $\times R R M 2$ & -0.27 & 0.33 \\
\hline$R R M 1 \times R R M 2 \times C D A$ & -0.28 & 0.31 \\
\hline$h E N T 1 \times d C K / R R M 1 \times R R M 2 \times C D A$ & -0.21 & 0.44 \\
\hline
\end{tabular}

${ }^{\mathrm{a}} \mathrm{P}<0.05$

Table VI. Correlation between the $\mathrm{IC}_{50}$ values for 5 -FU and the mRNA expression levels of genes associated with 5-FU metabolism.

\begin{tabular}{|c|c|c|}
\hline Gene expressions & $\begin{array}{l}\text { Spearman's rank- } \\
\text { correlation coefficient (Q) }\end{array}$ & P-value \\
\hline$T P$ & 0.064 & 0.82 \\
\hline$D P D$ & 0.37 & 0.17 \\
\hline OPRT & 0.086 & 0.76 \\
\hline$T S$ & 0.41 & 0.12 \\
\hline$T P / D P D$ & -0.38 & 0.16 \\
\hline$O P R T / D P D$ & -0.44 & 0.10 \\
\hline$T P \times O P R T$ & 0.043 & 0.88 \\
\hline$T S \times D P D$ & 0.55 & $0.035^{\mathrm{a}}$ \\
\hline$T P \times O P R T / T S \times D P D$ & -0.48 & 0.074 \\
\hline
\end{tabular}

${ }^{\mathrm{a}} \mathrm{P}<0.05$

catalyze another process that yields 5'-fluoro-2'-deoxyuridine5'-monophosphate, which forms a stable ternary complex with TS to block DNA synthesis and repair (40), there was no significant correlation between the $\mathrm{IC}_{50}$ values for 5 -FU and the expression levels of RRM1 or RRM2.

Establishment of GEM-resistant pancreatic cancer cells. To investigate the altered expression levels of GEM transport- and metabolism-related genes in GEM-resistant cells, the GEMresistant pancreatic cancer cells SUIT-2-GR and Capan-1-GR were generated from the parental cell lines (SUIT-2-parent and Capan-1-parent). The GEM IC ${ }_{50}$ values for both of these GEM-resistant cell lines were significantly higher than those of the parental cells (Table VII, Fig. $2 \mathrm{~A}$ and $\mathrm{B}$; $\mathrm{P}<0.001$ ).
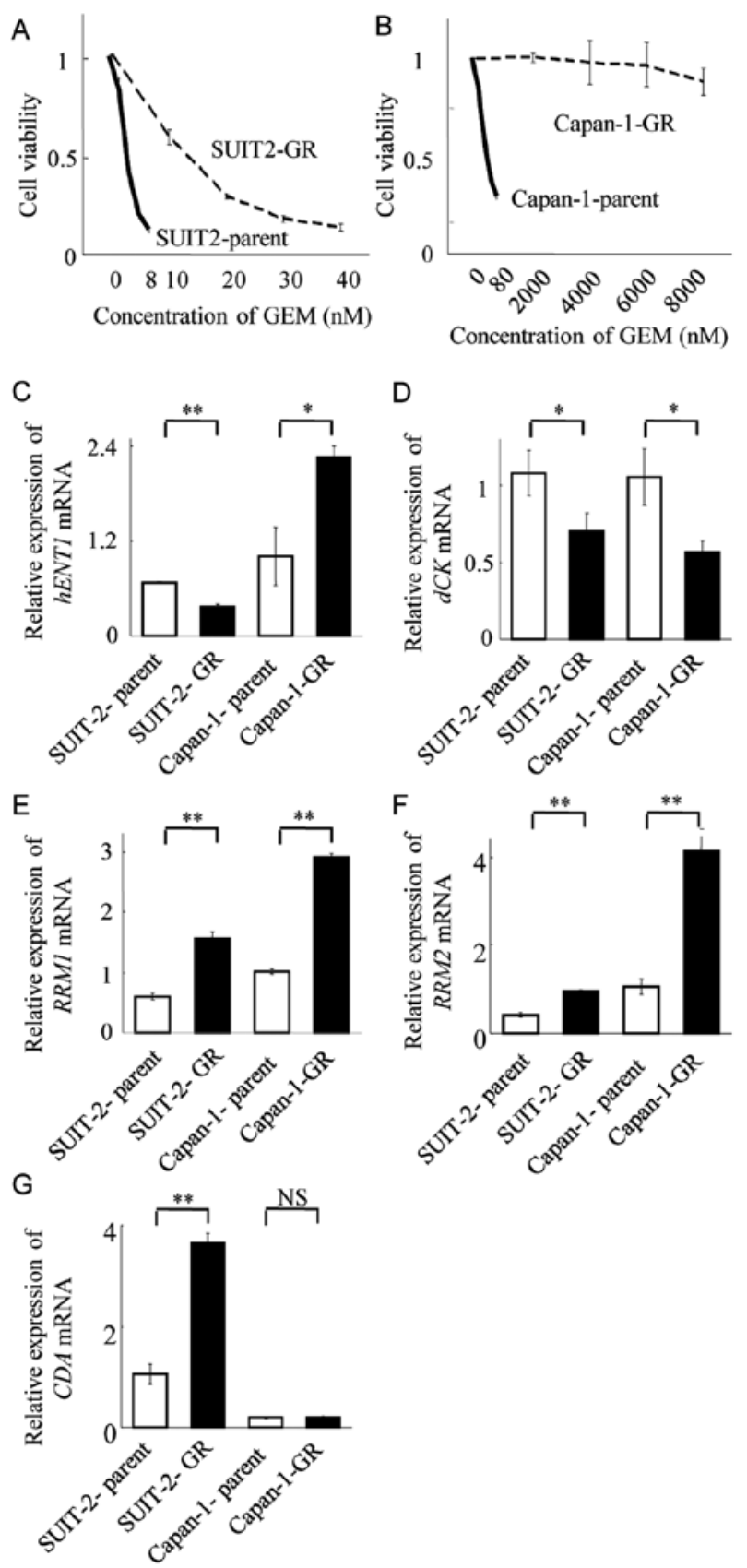

Figure 2. Viability of parental and GEM-resistant cells (SUIT-2-GR and Capan-1-GR) exposed to GEM (A and B). Both GEM-resistant cell lines were significantly more resistant to GEM than the parental cells. Quantitative analyses of $h E N T 1$ (C), $d C K(\mathrm{D}), R R M 1$ (E), RRM2 (F) and $C D A(\mathrm{G})$ mRNAs in parental and GEM-resistant cells (SUIT-2-GR and Capan-1-GR). ${ }^{*} \mathrm{P}<0.05 ;{ }^{* *} \mathrm{P}<0.01 ; \mathrm{NS}$, not significant.

Although SUIT-2-GR cells showed significantly decreased expression levels of $h E N T 1$, Capan-1-GR cells showed a significant increase in expression (Fig. 2C). The expression levels of $d C K$ significantly decreased (Fig. 2D) and those of $R R M 1$ and $R R M 2$ significantly increased in both of the GEM-resistant cell lines (Fig. 2E and F). The expression level of $C D A$ in SUIT-2-GR cells was significantly higher than that in SUIT-2-parent cells, whereas expressions in the Capan-1parent and Capan-1-GR cells were very low (and not significantly different) (Fig. 2G). The data regarding the expression levels of RRMI in GEM-resistant cells were 
Table VII. IC $_{50}$ values of GEM-resistant, 5-FU-resistant and parental cell lines.

\begin{tabular}{lcc}
\hline & \multicolumn{2}{c}{$\mathrm{IC}_{50}$ value } \\
\cline { 2 - 3 } Cell line & $\mathrm{GEM}(\mathrm{nM})$ & 5 -FU $(\mu \mathrm{M})$ \\
\hline SUIT-2-parent & $3.53 \pm 0.062$ & $4.01 \pm 0.17$ \\
SUIT-2-GR & $12.24 \pm 1.07$ & $2.12 \pm 0.15$ \\
SUIT-2-FR & $3.03 \pm 0.096$ & $7.33 \pm 0.24$ \\
Capan-1-parent & $62.63 \pm 6.86$ & $2.35 \pm 0.24$ \\
Capan-1-GR & $>8000$ & $0.78 \pm 0.17$ \\
Capan-1-FR & $2.69 \pm 0.13$ & $7.27 \pm 0.63$ \\
\hline
\end{tabular}

consistent with the above results in all 15 pancreatic cancer cell lines. Although previous reports show that decreased expression of $h E N T 1$ (20) and increased expression of $C D A$ (16), which were only observed in SUIT-2-GR cells, is associated with the development of GEM-resistance, our results from both of the GEM-resistant cells indicated that lower $d C K$, expression, coupled with higher $R R M 1$ and $R R M 2$ expressions, are important factors for developing resistance to this agent.

Establishment of 5-FU-resistant pancreatic cancer cells. We generated 5-FU-resistant SUIT-2 (SUIT-2-FR) and Capan-1 (Capan-1-FR) cells by exposure to gradually increasing concentrations of 5-FU. The 5-FU IC ${ }_{50}$ values for both of these resistant cell lines were significantly higher than those of the parental cell lines (Table VII and Fig. 3A and B; P<0.001). As outlined above for the GEM-resistance cells, we measured the expression of genes associated with 5-FU metabolism (Fig. 3C-F). The expression levels of $T P, D P D, O P R T$ and $T S$ in SUIT-2-FR cells were significantly higher than those in the parental cells (Fig. 3C-F). Meanwhile, Capan-1-FR cells showed a significant increase in $T P$ expression compared with Capan-1-parent cells (Fig. 3C). Although the level of DPD expression in Capan-1-FR cells was significantly higher than that in Capan-1-parent cells, it was still extremely low (Fig. 3D) compared with that in the SUIT-2 cell lines. The expression levels of $O P R T$ and TS in both of the Capan-1 cell lines were almost the same (Fig. 3E and F). Although previous reports showed that increased expressions of $O P R T$ and $T S$, which we observed only in SUIT-2-FR cells, were associated with resistance to 5-FU and its prodrugs $(32,38,40)$, our results from both of the 5-FU-resistant cell lines indicated that increased expressions of $T P$ and $D P D$ are important for developing 5-FU-resistance. We also calculated the $T S$ x $D P D$ expression level in these cell lines and found that both 5-FU-resistant cell lines showed significantly higher levels of combined expression than the parental cells, although expression levels in Capan-1 cells were lower than those in SUIT-2 cells (Fig. 3G).

GEM-sensitivity of 5-FU-resistant pancreatic cancer cells. To investigate whether there was any cross-resistance to $5-\mathrm{FU}$ and GEM, we examined the sensitivity of 5-FU-resistant cells to GEM. The GEM IC $\mathrm{I}_{50}$ value of SUIT-2-FR cells was slightly (but significantly) lower than that of the parental cells (Fig. 4A
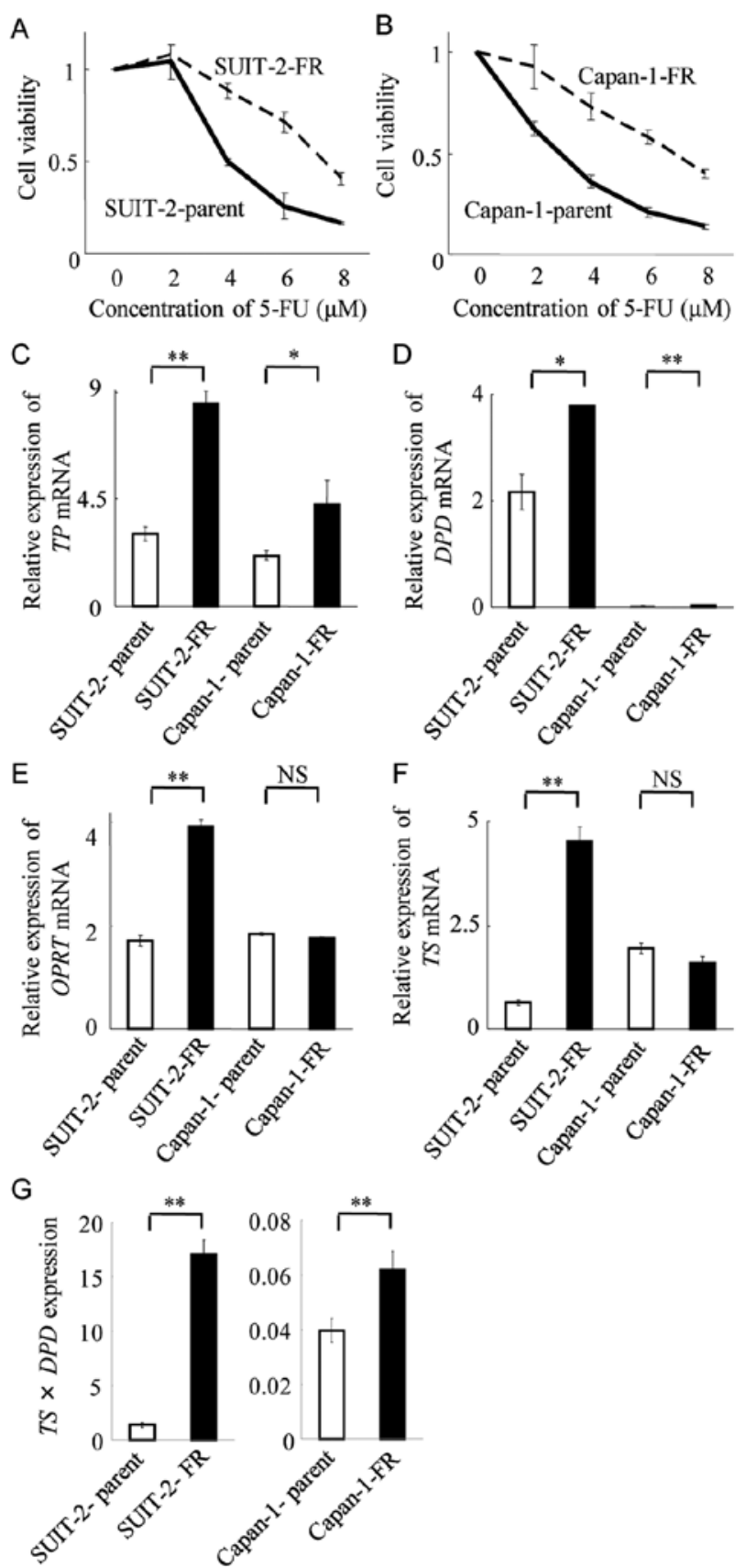

Figure 3. Viability of parental and 5-FU-resistant cells (SUIT-2-FR and Capan-1-FR) exposed to 5-FU (A and B). Both 5-FU-resistant cell lines were significantly more resistant to 5-FU than the parental cells. Quantitative analyses of TP (C), DPD (D), OPRT (E) and TS (F) mRNAs in parental and 5-FU-resistant cells (SUIT-2-FR and Capan-1-FR). Combined expression of $T S$ x DPD in parental and 5-FU-resistant cells (G). ${ }^{*} \mathrm{P}<0.05 ;{ }^{* *} \mathrm{P}<0.01$; NS, not significant.

and Table IV; $\mathrm{P}<0.001)$; however, there was no significant difference in GEM-sensitivity between Capan-1-FR cells and Capan-1-parent cells (Fig. 4B). This suggests that the acquisition of 5-FU-resistance had no effect on GEM-sensitivity.

To assess the effects of 5-FU-resistance on the expression levels of genes related to GEM transport and metabolism, we measured the expression levels of these genes in 5-FU-resistant and parental cells. SUIT-2-FR cells expressed significantly higher levels of $h E N T 1, d C K, R R M 1$ and RRM2, and significantly lower levels of $C D A$, than the parental cells (Fig. 4C-G). 

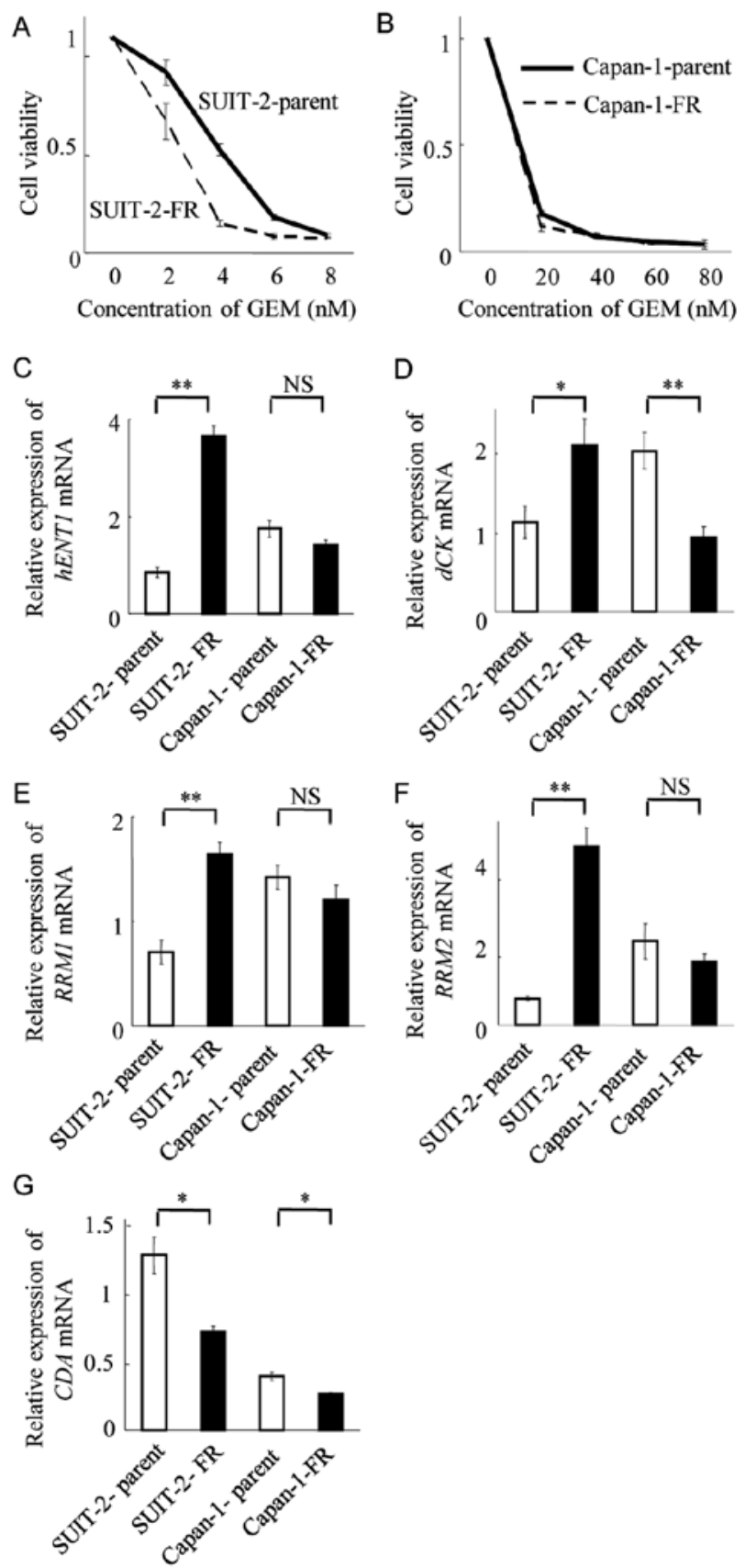

Figure 4. Viability of parental and 5-FU-resistant cells (SUIT-2-FR and Capan-1-FR) exposed to GEM (A and B). SUIT-2-FR cells were slightly, but significantly, more sensitive than SUIT-2-parent cells (A). Quantitative analyses of $h E N T 1$ (C), $d C K$ (D), RRM1 (E), RRM2 (F) and $C D A(\mathrm{G})$ mRNAs in parental and 5-FU-resistant cells (SUIT-2-FR and Capan-1-FR). ${ }^{*} \mathrm{P}<0.05 ;{ }^{* *} \mathrm{P}<0.01 ; \mathrm{NS}$, not significant.

Meanwhile, Capan-1-FR cells showed no significant changes in expression levels of $h E N T 1, R R M 1$ and RRM2 compared with the parental cells (Fig. 4C, E and F), although they did show significantly decreased expressions of $d C K$ and $C D A$ (Fig. 4D and G). Despite significantly increased expressions of $R R M 1$ and $R R M 2$, SUIT-2-FR cells did not become resistant to GEM. These results suggest that there may be a substantial number of patinets who become sensitive to GEM (via increased expressions of $d C K$ and $h E N T 1$ ) after developing resistance to 5-FU.
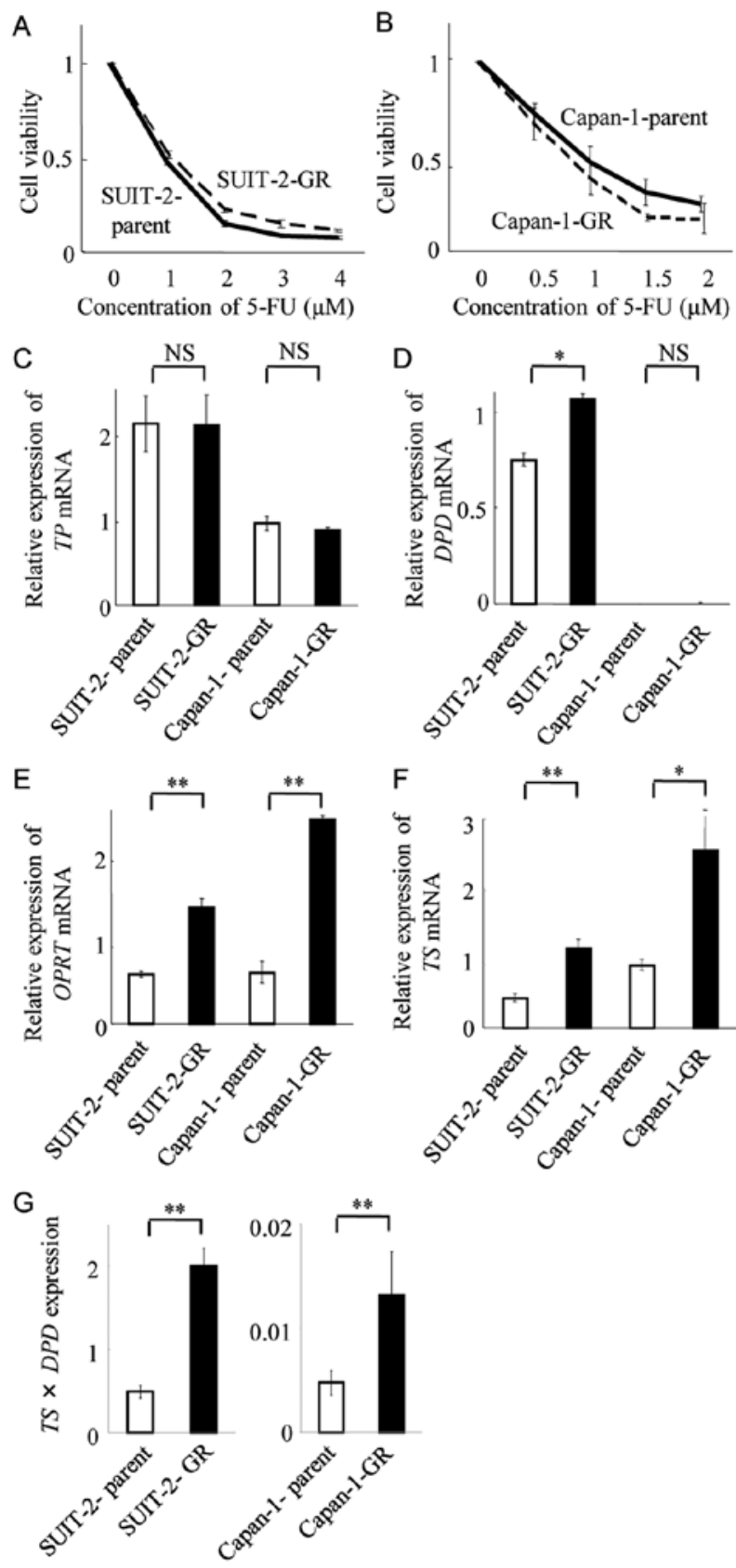

Figure 5. Viability of parental and GEM-resistant cells (SUIT-2-GR and Capan-1-GR) exposed to 5-FU (A and B). Quantitative analyses of TP (C), $D P D(\mathrm{D}), O P R T$ (E) and TS (F) mRNAs in parental and GEM-resistant cells (SUIT-2-GR and Capan-1-GR). Combined expression of TS x DPD in parental and GEM-resistant cells $(\mathrm{G}) .{ }^{*} \mathrm{P}<0.05 ;{ }^{* * *} \mathrm{P}<0.01$; NS, not significant.

5-FU sensitivity of GEM-resistant pancreatic cancer cells. We also investigated the sensitivity to 5-FU of GEM-resistant cells and found that GEM-resistant cells had similar levels of 5-FU-sensitivity to the parental cells (Fig. 5A and B). These data suggest that GEM-resistance did not affect 5-FUsensitivity.

Similarly, we measured the expression levels of the genes related to 5-FU metabolism in GEM-resistant cell lines (Fig. 5C-F). Although there was no significant change in the expression level of $T P$, the levels of $O P R T, T S$ and $T S$ x $D P D$ expression were significantly higher in both of the GEMresistant cell lines than in parental cells (Fig. 5C, E-G). 
SUIT-2-GR cells showed significantly higher level of $D P D$ expression than the SUIT-2-parent cells, whereas the expression levels in Capan-1-GR and Capan-1-parent cells were too low to compare (Fig. 5D). Although GEM-resistant cells showed significant increases in $D P D, O P R T, T S$ and $T S$ x DPD expression levels (as observed in 5-FU-resistant cells) (Fig. 3C-G), they did not become resistant to 5-FU. This suggests that increased expression of $T P$ may be essential for the development of 5-FU-resistance in both cell lines.

\section{Discussion}

Although GEM-based chemotherapy is still the standard palliative chemotherapy for pancreatic cancer $(5,11)$, the efficacy of GEM as a single agent is limited, and clinicians are often torn when faced with GEM-refractory patients. To improve the prognosis of patients with pancreatic cancer, much effort has been put into developing other effective firstand second-line chemotherapy regimens such as 5-FU prodrugs, used alone or in combination with GEM; however, their therapeutic effects are modest or disappointing. Therefore, individualized chemotherapy based on the gene expression profiles of the individual's own cancer tissues would be a helpful strategy for selecting those patients that are likely to respond to treatment $(20,33)$. Many studies of the mechanisms of GEM and 5-FU metabolism have suggested that certain genes/proteins are associated with sensitivity to these drugs $(12-15,17-26,39,40)$. However, to our knowledge, there is no study evaluating acquired cross-resistance between GEM and 5-FU and its correlation with gene expression.

In the present study, we analyzed the $\mathrm{IC}_{50}$ values for GEM and 5-FU in 15 pancreatic cancer cell lines and found no correlation between sensitivity to either drug. Moreover, we evaluated sensitivity to these agents using pancreatic cancer cell lines resistant to either GEM or 5-FU and found that these GEM- or 5-FU-resistant cells acquired no cross-resistance to the other agent. These data suggest that first line chemotherapy using either GEM- or 5-FU may not promote resistance to the other drug and confirm that combination therapy, or secondline chemotherapy using one or other of the drugs, may be a useful strategy for treating pancreatic cancer. Notably, SUIT2-FR cells showed slightly (but significantly) higher sensitivity to GEM than parental cells. However, recent clinical studies have not shown striking results with second-line chemotherapy (41-43); therefore, further investigation is needed to select the best agents for first- or second-line chemotherapy for pancreatic cancer.

To evaluate whether any changes occurred in cells that developed resistance to GEM or 5-FU, we also analyzed the expression levels of the genes associated with transport and metabolism in 15 pancreatic cancer cell lines and GEM- or 5-FU resistant cells. Regarding GEM, the present data suggest that lower expression of $d C K$, coupled with higher expressions of $R R M 1$ and $R R M 2$ may be important factors for developing the resistance to this drug. Akita et al (44) demonstrated that only patients with low levels of RRM1 expression derive significant benefit from GEM in terms of preventing disease recurrence. Therefore, RRM1 expression may contribute to GEM-resistance in pancreatic cancer. However, SUIT-2-FR cells did not become resistant to GEM, despite increased
RRMI expression levels, suggesting that quantification of several genes and a combined evaluation of the results may be needed if individualized chemotherapy based on gene expression profiles is to be used in a clinical setting.

The data regarding 5-FU-resistant cells suggest that higher $T P, D P D$ and $T S \times D P D$ expressions may be important factors for developing the resistance to this drug. There was no significant change in 5-FU-sensitivity in GEM-resistant cells expressing higher levels of $D P D, O P R T, T S$ and $T S \times D P D$, suggesting that increased expression levels of $T P$ may be essential for the development of 5-FU-resistance. Increased $T P$ expression was initially reported to be correlated with increased sensitivity to $5-\mathrm{FU}$, possibly due to increased synthesis of 2'-deoxy-5'-fluorouridine (FUDR) (45). However, higher TP expression was also reported to correlate with a poor response to 5-FU-based treatment and shorter survival times in colorectal (45) and pancreatic cancer (39) patients, although there are conflicting results (46). TP is identical to platelet-derived endothelial cell growth factor (PD-ECGF) in terms of its pro-angiogenic activity; therefore, the activity of this enzyme is used as a prognostic indicator (47). Conversely, TP is also an enzyme that metabolizes the 5-FU prodrug, capecitabine (N4-pentoxycarbonyl-5'-5-fluorocytidine). This is an attractive novel fluoropyrimidine analogue with great clinical potential. It is metabolized in the liver and tumor tissues to 5'-deoxy-5fluorouridine (5'-DFUR) by CDA. 5'-DFUR is then converted to 5 -FU by TP $(26,46)$. Because TP is highly expressed in tumor tissues relative to host cells, capecitabine can be selectively activated in tumor tissues, suggesting that TP may contribute to capecitabine sensitivity $(26,31)$. Additionally, CDA is also associated with GEM-resistance due to its ability to inactivate GEM (48). Therefore, capecitabine may be a potent drug for treating GEM-resistant patients showing high $C D A$ expression, or for 5-FU-resistant patients showing high $T P$ expression. However, further studies are needed to elucidate the correlation between capecitabine-sensitivity and $C D A$ and/or $T P$ expression.

In conclusion, we found no cross-resistance between GEM and 5-FU, even in pancreatic cancer cell lines that developed resistance to the other drug. These results suggest that it may be possible to use either of these drugs as second-line chemotherapy in patients with pancreatic cancer that has developed resistance to one of these agents. In addition, quantitative analyses of RRM1, TP, DPD and TS may be a potent strategy for developing individualized chemo-therapeutic regimens.

\section{Acknowledgments}

We thank Ms. S. Sadatomi, Ms. M. Sato, Ms. M. Ohmori, Ms. E. Manabe, and Ms. M. Masuda (Department of Surgery and Oncology, Kyushu University Hospital) for skillful examination and analysis. This work was supported, in part, by a grant-in-aid from the Ministry of Education, Culture, Sports, Science and Technology of Japan, and by grants from the Japanese Society of Gastroenterology, the Pancreas Research Foundation of Japan, and the Kaibara Morikazu Medical Science Promotion Foundation of Japan.

\section{References}

1. Li D, Xie K, Wolff R and Abbruzzese JL: Pancreatic cancer. Lancet 363: 1049-1057, 2004. 
2. Matsuno S, Egawa S, Fukuyama S, et al: Pancreatic Cancer Registry in Japan: 20 years of experience. Pancreas 28: 219-230, 2004.

3. Carpelan-Holmstrom M, Nordling S, Pukkala E, et al: Does anyone survive pancreatic ductal adenocarcinoma? A nationwide study re-evaluating the data of the Finnish Cancer Registry. Gut 54: 385-387, 2005.

4. Wagner M, Redaelli C, Lietz M, Seiler CA, Friess H and Buchler MW: Curative resection is the single most important factor determining outcome in patients with pancreatic adenocarcinoma. Br J Surg 91: 586-594, 2004.

5. Burris HA 3rd, Moore MJ, Andersen J, et al: Improvements in survival and clinical benefit with gemcitabine as first-line therapy for patients with advanced pancreas cancer: a randomized trial. J Clin Oncol 15: 2403-2413, 1997.

6. Colucci G, Labianca R, Di Costanzo F, et al: Randomized phase III trial of gemcitabine plus cisplatin compared with single-agent gemcitabine as first-line treatment of patients with advanced pancreatic cancer: the GIP-1 study. J Clin Oncol 28: 1645-1651, 2010.

7. Cunningham D, Chau I, Stocken DD, et al: Phase III randomized comparison of gemcitabine versus gemcitabine plus capecitabine in patients with advanced pancreatic cancer. J Clin Oncol 27 5513-5518, 2009

8. Kindler HL, Niedzwiecki D, Hollis D, et al: Gemcitabine plus bevacizumab compared with gemcitabine plus placebo in patients with advanced pancreatic cancer: phase III trial of the cancer and leukemia group B (CALGB 80303). J Clin Oncol 28 : 3617-3622, 2010

9. Moore MJ, Goldstein D, Hamm J, et al: Erlotinib plus gemcitabine compared with gemcitabine alone in patients with advanced pancreatic cancer: a phase III trial of the Nationa Cancer Institute of Canada Clinical Trials Group. J Clin Oncol 25: 1960-1966, 2007.

10. Carmichael J: The role of gemcitabine in the treatment of other tumours. Br J Cancer 78 (Suppl 3): S21-S25, 1998.

11. Oettle H, Post S, Neuhaus P, et al: Adjuvant chemotherapy with gemcitabine vs observation in patients undergoing curativeintent resection of pancreatic cancer: a randomized controlled trial. JAMA 297: 267-277, 2007.

12. Garcia-Manteiga J, Molina-Arcas M, Casado FJ, Mazo A and Pastor-Anglada M: Nucleoside transporter profiles in human pancreatic cancer cells: role of hCNT1 in 2',2'-difluorodeoxycytidine-induced cytotoxicity. Clin Cancer Res 9: 5000-5008, 2003.

13. Fukunaga AK, Marsh S, Murry DJ, Hurley TD and McLeod HL: Identification and analysis of single-nucleotide polymorphisms in the gemcitabine pharmacologic pathway. Pharmacogenomics J 4: 307-314, 2004

14. Plunkett W, Huang P, Searcy CE and Gandhi V: Gemcitabine: preclinical pharmacology and mechanisms of action. Semin Oncol 23: 3-15, 1996.

15. Heinemann V, Xu YZ, Chubb S, et al: Cellular elimination of 2',2'-difluorodeoxycytidine 5'-triphosphate: a mechanism of selfpotentiation. Cancer Res 52: 533-539, 1992.

16. Eda H, Ura M, K FO, Tanaka Y, Miwa M and Ishitsuka H: The antiproliferative activity of DMDC is modulated by inhibition of cytidine deaminase. Cancer Res 58: 1165-1169, 1998.

17. Blackstock AW, Lightfoot H, Case LD, et al: Tumor uptake and elimination of 2',2'-difluoro-2'-deoxycytidine (gemcitabine) after deoxycytidine kinase gene transfer: correlation with in vivo tumor response. Clin Cancer Res 7: 3263-3268, 2001.

18. Duxbury MS, Ito H, Zinner MJ, Ashley SW and Whang EE: RNA interference targeting the M2 subunit of ribonucleotide reductase enhances pancreatic adenocarcinoma chemosensitivity to gemcitabine. Oncogene 23: 1539-1548, 2004.

19. Fujita H, Ohuchida K, Mizumoto K, et al: Gene expression levels as predictive markers of outcome in pancreatic cancer after gemcitabine-based adjuvant chemotherapy. Neoplasia 12 : $807-817,2010$

20. Giovannetti E, Del Tacca M, Mey V, et al: Transcription analysis of human equilibrative nucleoside transporter-1 predicts survival in pancreas cancer patients treated with gemcitabine. Cancer Res 66: 3928-3935, 2006.

21. Mackey JR, Mani RS, Selner M, et al: Functional nucleoside transporters are required for gemcitabine influx and manifestation of toxicity in cancer cell lines. Cancer Res 58 4349-4357, 1998.

22. Nakahira S, Nakamori S, Tsujie M, et al: Involvement of ribonucleotide reductase M1 subunit overexpression in gemcitabine resistance of human pancreatic cancer. Int J Cancer 120: $1355-1363,2007$.
23. Ohhashi S, Ohuchida K, Mizumoto K, et al: Down-regulation of deoxycytidine kinase enhances acquired resistance to gemcitabine in pancreatic cancer. Anticancer Res 28: 2205-2212, 2008.

24. Rosell R, Felip E, Taron M, et al: Gene expression as a predictive marker of outcome in stage IIB-IIIA-IIIB non-small cell lung cancer after induction gemcitabine-based chemotherapy followed by resectional surgery. Clin Cancer Res 10: 4215-4219, 2004.

25. Spratlin J, Sangha R, Glubrecht D, et al: The absence of human equilibrative nucleoside transporter 1 is associated with reduced survival in patients with gemcitabine-treated pancreas adenocarcinoma. Clin Cancer Res 10: 6956-6961, 2004.

26. Guichard SM, Macpherson JS, Mayer I, et al: Gene expression predicts differential capecitabine metabolism, impacting on both pharmacokinetics and antitumour activity. Eur J Cancer 44: 310-317, 2008.

27. Okusaka T, Funakoshi A, Furuse J, et al: A late phase II study of S-1 for metastatic pancreatic cancer. Cancer Chemother Pharmacol 61: 615-621,2008.

28. Shirasaka T, Shimamato Y, Ohshimo H, et al: Development of a novel form of an oral 5-fluorouracil derivative (S-1) directed to the potentiation of the tumor selective cytotoxicity of 5-fluorouracil by two biochemical modulators. Anticancer Drugs 7: 548-557, 1996.

29. Tatsumi K, Fukushima M, Shirasaka T and Fujii S: Inhibitory effects of pyrimidine, barbituric acid and pyridine derivatives on 5-fluorouracil degradation in rat liver extracts. Jpn J Cancer Res 78: 748-755, 1987.

30. Shirasaka T, Shimamoto $\mathrm{Y}$ and Fukushima M: Inhibition by oxonic acid of gastrointestinal toxicity of 5-fluorouracil without loss of its antitumor activity in rats. Cancer Res 53: 4004-4009, 1993.

31. Ishikawa T, Sekiguchi F, Fukase $\mathrm{Y}$, Sawada $\mathrm{N}$ and Ishitsuka H: Positive correlation between the efficacy of capecitabine and doxifluridine and the ratio of thymidine phosphorylase to dihydropyrimidine dehydrogenase activities in tumors in human cancer xenografts. Cancer Res 58: 685-690, 1998.

32. Kuramochi H, Hayashi K, Uchida K, et al: High intratumoral dihydropyrimidine dehydrogenase mRNA levels in pancreatic cancer associated with a high rate of response to S-1. Cancer Chemother Pharmacol 63: 85-89, 2008.

33. Danesi R, De Braud F, Fogli S, Di Paolo A and Del Tacca M: Pharmacogenetic determinants of anti-cancer drug activity and toxicity. Trends Pharmacol Sci 22: 420-426, 2001.

34. Sato N, Mizumoto K, Beppu K, et al: Establishment of a new human pancreatic cancer cell line, NOR-P1, with high angiogenic activity and metastatic potential. Cancer Lett 155: 153-161, 2000.

35. Ohuchida K, Mizumoto K, Murakami M, et al: Radiation to stromal fibroblasts increases invasiveness of pancreatic cancer cells through tumor-stromal interactions. Cancer Res 64: 3215-3222, 2004.

36. Ohuchida K, Mizumoto K, Ogura Y, et al: Quantitative assessment of telomerase activity and human telomerase reverse transcriptase messenger RNA levels in pancreatic juice samples for the diagnosis of pancreatic cancer. Clin Cancer Res 11: 2285-2292, 2005.

37. Nakano Y, Tanno S, Koizumi K, et al: Gemcitabine chemoresistance and molecular markers associated with gemcitabine transport and metabolism in human pancreatic cancer cells. Br J Cancer 96: 457-463, 2007

38. Ichikawa W, Uetake H, Shirota Y, et al: Both gene expression for orotate phosphoribosyltransferase and its ratio to dihydropyrimidine dehydrogenase influence outcome following fluoropyrimidine-based chemotherapy for metastatic colorectal cancer. Br J Cancer 89: 1486-1492, 2003.

39. Miyake K, Imura S, Yoshizumi T, Ikemoto T, Morine Y and Shimada M: Role of thymidine phosphorylase and orotate phosphoribosyltransferase mRNA expression and its ratio to dihydropyrimidine dehydrogenase in the prognosis and clinicopathological features of patients with pancreatic cancer. Int $J$ Clin Oncol 12: 111-119, 2007

40. Allen WL and Johnston PG: Role of genomic markers in colorectal cancer treatment. J Clin Oncol 23: 4545-4552, 2005.

41. Androulakis N, Syrigos K, Polyzos A, et al: Oxaliplatin for pretreated patients with advanced or metastatic pancreatic cancer: a multicenter phase II study. Cancer Invest 23: 9-12, 2005.

42. Demols A, Peeters M, Polus M, et al: Gemcitabine and oxaliplatin (GEMOX) in gemcitabine refractory advanced pancreatic adenocarcinoma: a phase II study. Br J Cancer 94: 481-485, 2006. 
43. Kulke MH, Blaszkowsky LS, Ryan DP, et al: Capecitabine plus erlotinib in gemcitabine-refractory advanced pancreatic cancer. J Clin Oncol 25: 4787-4792, 2007.

44. Akita H, Zheng Z, Takeda Y, et al: Significance of RRM1 and ERCC1 expression in resectable pancreatic adenocarcinoma. Oncogene 28: 2903-2909, 2009.

45. Metzger R, Danenberg K, Leichman CG, et al: High basal leve gene expression of thymidine phosphorylase (platelet-derived endothelial cell growth factor) in colorectal tumors is associated with nonresponse to 5-fluorouracil. Clin Cancer Res 4: 2371-2376, 1998.
46. Ackland SP and Peters GJ: Thymidine phosphorylase: its role in sensitivity and resistance to anticancer drugs. Drug Resist Updat 2: 205-214, 1999

47. Ishikawa F, Miyazono K, Hellman U, et al: Identification of angiogenic activity and the cloning and expression of plateletderived endothelial cell growth factor. Nature 338: 557-562, 1989.

48. Bengala C, Guarneri V, Giovannetti E, et al: Prolonged fixed dose rate infusion of gemcitabine with autologous haemopoietic support in advanced pancreatic adenocarcinoma. Br J Cancer 93: 35-40, 2005. 